\title{
Gene therapy enhances chemotherapy tolerance and efficacy in glioblastoma patients
}

\author{
Jennifer E. Adair, ${ }^{1,2}$ Sandra K. Johnston, ${ }^{3}$ Maciej M. Mrugala, ${ }^{4,5}$ Brian C. Beard, ${ }^{1,2}$ Laura A. Guyman, ${ }^{6,7}$ Anne L. Baldock, \\ Carly A. Bridge, ${ }^{6,7}$ Andrea Hawkins-Daarud, ${ }^{6,7}$ Jennifer L. Gori, ${ }^{1}$ Donald E. Born, ${ }^{8}$ Luis F. Gonzalez-Cuyar, ${ }^{9}$ Daniel L. Silbergeld, ${ }^{3,9}$ \\ Russell C. Rockne, ${ }^{6,7}$ Barry E. Storer, ${ }^{1,10}$ Jason K. Rockhill, ${ }^{3,11}$ Kristin R. Swanson, ${ }^{6,7,12}$ and Hans-Peter Kiem ${ }^{1,2,9}$ \\ ${ }^{1}$ Clinical Research Division, Fred Hutchinson Cancer Research Center, Seattle, Washington, USA. ${ }^{2}$ Department of Medicine, ${ }^{3}$ Department of Radiology, ${ }^{4}$ Department of Neurosurgery, and ${ }^{5}$ Department of \\ Neurology, University of Washington (UW), Seattle, Washington, USA. ${ }^{6}$ Department of Neurological Surgery and ${ }^{7}$ Northwestern Brain Tumor Institute, Northwestern University Feinberg School of Medicine, \\ Chicago, Illinois, USA. ${ }^{8}$ Department of Pathology, Stanford University, Stanford, California, USA. ${ }^{9}$ Department of Pathology, ${ }^{10}$ Department of Biostatistics, and ${ }^{11}$ Department of Radiation Oncology, UW, \\ Seattle, Washington, USA. ${ }^{12}$ Robert H. Lurie Comprehensive Cancer Center, Northwestern University Feinberg School of Medicine, Chicago, Illinois, USA
}

\begin{abstract}
BACKGROUND. Temozolomide (TMZ) is one of the most potent chemotherapy agents for the treatment of glioblastoma. Unfortunately, almost half of glioblastoma tumors are TMZ resistant due to overexpression of methylguanine methyltransferase (MCMThi). Coadministration of $\mathrm{O}^{6}$-benzylguanine $\left(\mathrm{O}^{6} \mathrm{BC}\right)$ can restore TMZ sensitivity, but causes off-target myelosuppression. Here, we conducted a prospective clinical trial to test whether gene therapy to confer $\mathrm{O}^{6} \mathrm{BC}$ resistance in hematopoietic stem cells (HSCs) improves chemotherapy tolerance and outcome.
\end{abstract}

METHODS. We enrolled 7 newly diagnosed glioblastoma patients with MGMThi tumors. Patients received autologous gene-modified HSCs following single-agent carmustine administration. After hematopoietic recovery, patients underwent $0^{6} \mathrm{BC} / \mathrm{TMZ}$ chemotherapy in 28-day cycles. Serial blood samples and tumor images were collected throughout the study. Chemotherapy tolerance was determined by the observed myelosuppression and recovery following each cycle. Patientspecific biomathematical modeling of tumor growth was performed. Progression-free survival (PFS) and overall survival (OS) were also evaluated.

RESULTS. Gene therapy permitted a significant increase in the mean number of tolerated $0^{6} \mathrm{BG} / \mathrm{TMZ}$ cycles (4.4 cycles per patient, $P<0.05$ ) compared with historical controls without gene therapy ( $n=7$ patients, 1.7 cycles per patient). One patient tolerated an unprecedented 9 cycles and demonstrated long-term PFS without additional therapy. Overall, we observed a median PFS of 9 (range 3.5-57+) months and OS of 20 (range 13-57+) months. Furthermore, biomathematical modeling revealed markedly delayed tumor growth at lower cumulative TMZ doses in study patients compared with patients that received standard $\mathrm{TMZ}$ regimens without $\mathrm{O}^{6} \mathrm{BC}$.

CONCLUSION. These data support further development of chemoprotective gene therapy in combination with $0^{6} \mathrm{BC}$ and $\mathrm{TMZ}$ for the treatment of glioblastoma and potentially other tumors with overexpression of MGMT.

TRIAL REGISTRATION. Clinicaltrials.gov NCT00669669.

FUNDING. R01CA114218, R01AI080326, R01HL098489, P30DK056465, K01DK076973, R01HL074162, R01CA164371, R01NS060752, U54CA143970.

\section{Introduction}

Glioblastoma multiforme (GBM), the most common and aggressive primary brain tumor in adults, is almost uniformly fatal, carrying a $47 \%$ chance of survival at one year from diagnosis and a median overall survival (OS) of 12 to 14 months (1). Unfortunately, over the past decade, new therapies in GBM have accomplished little to

Conflict of interest: The authors have declared that no conflict of interest exists Role of funding source: The content is solely the responsibility of the authors and does not necessarily represent the official views of the NIH nor its subsidiary Institutes and Centers.

Submitted: April 29, 2014; Accepted: July 1, 2014.

Reference information: J Clin Invest. 2014;124(9):4082-4092. doi:10.1172/JCI76739. improve OS. To date, the most effective chemotherapies to treat GBM are alkylating agents such as temozolomide (TMZ), which is the current standard chemotherapy regimen $(2,3)$. Despite TMZ potency, median survival in all GBM patients remains less than two years from diagnosis (median 21 months) (2, 3). Moreover, approximately $50 \%$ of GBM tumors overexpress methylguanine methyltransferase $\left(\mathrm{MGMT}^{\mathrm{hi}}\right)$, indicated by hypomethylation of the promoter controlling MGMT expression. MGMT ${ }^{\text {hi }}$ tumor cells are resistant to TMZ due to the ability of the catalytic MGMT protein to repair TMZ-induced cytotoxic DNA damage, conferring poor median survival (12.6 months) $(2,3)$. Notably, MGMT overexpression in alkylating agent-resistant tumor cells is also found in many other tumor types (reviewed in ref. 4). 
Presentation
Surgical Debulking/Resection

Diagnosis of WHO Grade IV GBM, screening of tumor tissue for MGMT promoter methylation (unmethylated $=$ eligible)

Screen Tests: $n=27$

Screen Failures: $n=12$

Patients Enrolled: $n=8$

Patients who Declined Enrollment: $n=7$
Weeks -8 to -2

Weeks -2 to -1

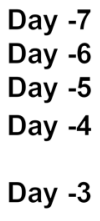

Day -3

Day -2

Day 0

After Hematopoietic Recovery from BCNU
Up to 24 cycles of $\mathrm{O}^{6} \mathrm{BG}+\mathrm{TMZ}$ $\left(472 \mathrm{mg} / \mathrm{m}^{2}\right), 28$ days in length, with optional $25 \% \mathrm{TMZ}$ dose escalation if $>1 \%$ gene modified granulocytes are present and at least two cycles were tolerated at lower TMZ dose
Pre-collection and transplant work-up; post-RT response evaluation (MRI or CT)

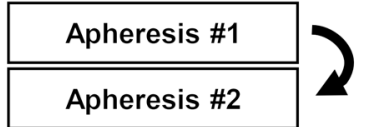

$2 \times 10^{6}$ unmanipulated $\mathrm{CD} 34^{+}$cells/kg body weight cryopreserved as backup $n=8$
Enrichment of $\geq 2.5 \times 10^{6}$ $\mathrm{CD} 34^{+}$cells per $\mathrm{kg}$ and subsequent gene transfer

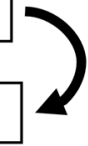

subsequent gene
$n=7$
Infusion of Gene-Modified Hematopoietic Stem Cells
Neurological exam each cycle

Response Evaluation (MRI or CT) every other cycle or if clinically indicated

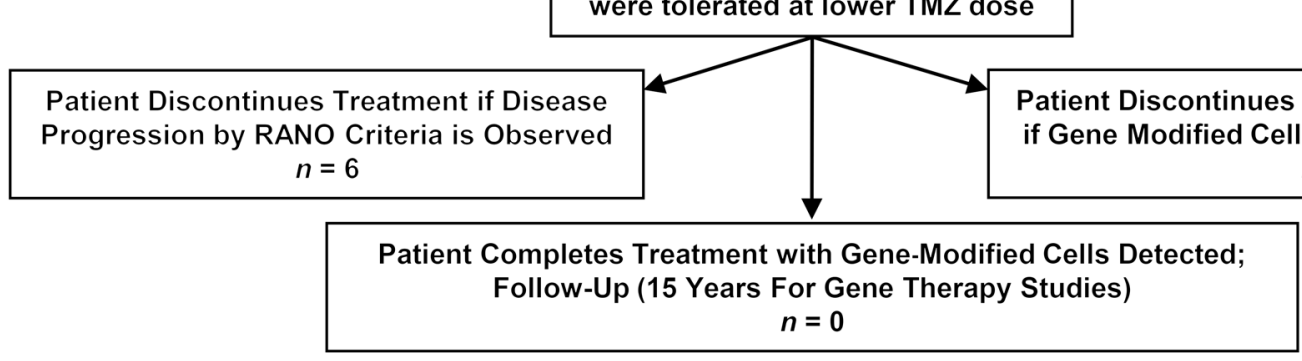

Figure 1. Flow diagram of study protocol.

The small nucleoside inhibitor, $\mathrm{O}^{6}$-benzylguanine $\left(\mathrm{O}^{6} \mathrm{BG}\right)$, effectively depletes MGMT activity by mimicking the methylated guanine nucleotide base targeted by MGMT, thereby binding MGMT protein and causing structural changes that mark the protein-nucleotide complex for degradation. Concurrent administration of $\mathrm{O}^{6} \mathrm{BG}$ restores tumor cell sensitivity to alkylating agents such as TMZ, which was previously investigated as a strategy to improve TMZ efficacy in recurrent GBM patients $(5,6)$. However, early phase studies revealed that $\mathrm{O}^{6} \mathrm{BG} / \mathrm{TMZ}$ administration caused severe off-target myelosuppression. Specifically, patients administered TMZ at the maximum tolerated dose (MTD) (472 $\mathrm{mg} / \mathrm{m}^{2}$ ) in combination with $\mathrm{O}^{6} \mathrm{BG}$ exhibited $46 \%$ incidence of grade 4 neutropenia $(5,6)$. The hematopoietic-specific toxicity was attributed to low-to-nonexistent levels of MGMT detected in hematopoietic stem cells (HSCs) and progenitor cells (7).
Previously, we and others have shown that expression of the $\mathrm{O}^{6} \mathrm{BG}$-resistant $M G M T$ mutant $P 140 \mathrm{~K}$ by hematopoietic cells provides significant chemoprotection against hematopoietic toxicity from $\mathrm{O}^{6} \mathrm{BG} /$ alkylator chemotherapy (8). Based on our preclinical studies, we initiated a prospective phase I/II clinical trial in which newly diagnosed MGMThi ${ }^{\text {hBM }}$ patients were transplanted with autologous $P 140 \mathrm{~K}$ gene-modified hematopoietic $\mathrm{CD} 34^{+}$cells to prevent hematopoietic toxicity during combination $\mathrm{O}^{6} \mathrm{BG} / \mathrm{TMZ}$ chemotherapy (9). We recently reported the feasibility and safety of this approach based on observations in the first three patients enrolled on this trial (9). We have now treated a total of seven patients to date. Here, we report the efficacy of this approach, which includes increased chemotherapy tolerance and improved outcome in these patients. 
Table 1. Patient demographics, treatment, and survival

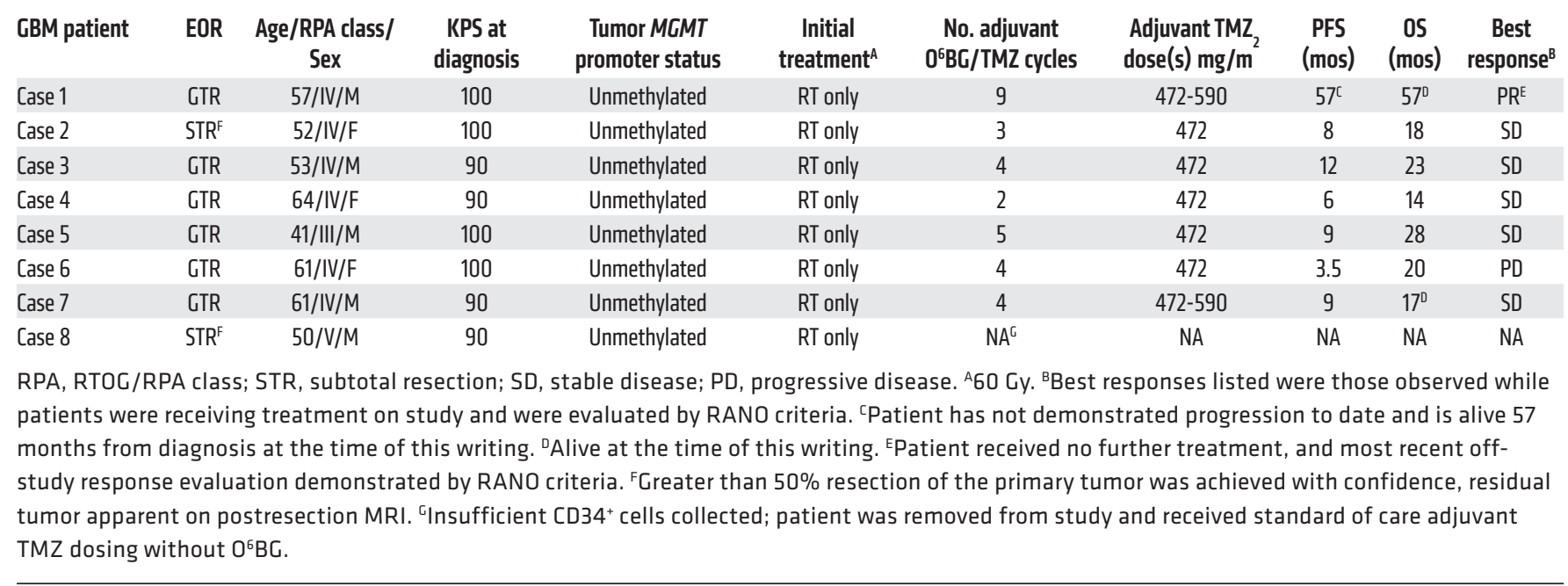

\section{Results}

Patient data and treatment. As detailed in Figure 1, a total of eight patients with newly diagnosed, MGMT promoter unmethylated malignant gliomas (WHO grade IV) were enrolled into the first phase of the study. One patient (patient 5) was classified as recursive partitioning analysis (RPA) class III (expected median survival 17.1 months, $70 \%$ OS rate at 1 year from diagnosis); five patients (patients 1, 2, 3, 4, 6, and 7) were classified as RPA class IV (expected median survival 11.2 months, $46 \% 1$ year OS rate); and one patient (case patient 8 ) was classified as RPA class V (expected median survival 7.5 months, 28\% 1 year OS rate) (Table 1 and ref. 10). All patients received a greater than $50 \%$ surgical resection of the primary tumor, followed by radiation therapy (RT) without concomitant TMZ, since this treatment is unlikely to significantly benefit patients with $M G M T$ promoter unmethylated tumors and would predictably compromise the HSC pool available for subsequent gene modification. Following RT, patients were mobilized with granulocyte colony-stimulating factor (G-CSF) and underwent leukapheresis; autologous peripheral blood CD $34^{+}$cells from each patient were enriched. One patient (case patient 8) did not sufficiently mobilize to allow for collection of $2.5 \times 10^{6}$ or more CD34 ${ }^{+}$ cells per kilogram $(\mathrm{kg})$ of body weight and was removed from the study. For all other patients, enriched CD $34^{+}$cells were genetically modified with a retroviral vector encoding the P140K transgene (Figure 2A). Following gene transfer, the observed mean level of colony-forming cells containing provirus was 65\% (range $41 \%$ $90 \%)$. Cell products were infused at doses ranging from $3.8 \times 10^{6}$ to $21.9 \times 10^{6} \mathrm{CD} 34^{+}$cells $/ \mathrm{kg}$ within 24 to 48 hours after administration of single-agent carmustine (BCNU) at a dose of $600 \mathrm{mg} / \mathrm{m}^{2}$. Following hematopoietic recovery from BCNU, study case patients received between 2 and 9 cycles (mean $=4.4$ cycles) of adjuvant chemotherapy, including $\mathrm{O}^{6} \mathrm{BG}$ and $\mathrm{TMZ}$ at or above the previously established MTD of TMZ $\left(472 \mathrm{mg} / \mathrm{m}^{2}\right)$ (Table 1). To maximize potential therapeutic benefit, intrapatient dose escalation of TMZ was permitted. Criteria for dose escalation required at least two prior cycles at the MTD of TMZ with hematopoietic recovery (absolute neutrophil count $[\mathrm{ANC}] \geq 1,500 / \mu \mathrm{l}$ and platelets $[\mathrm{PLTs}] \geq$
$100,000 / \mu \mathrm{l})$ within 42 days from the previous cycle and no grade 4 hematologic toxicity lasting longer than 7 days as well as no grade 4 nonhematologic toxicity associated with the therapy. Additionally, patients had to have had at least $1 \%$ detectable gene-modified granulocytes present in peripheral blood at the time of chemotherapy. Two enrolled patients (case patients 1 and 7) received a total of three TMZ dose-escalated cycles $\left(590 \mathrm{mg} / \mathrm{m}^{2}\right)$ based on these criteria. Patient response was measured every two months during treatment, and $\mathrm{O}^{6} \mathrm{BG} / \mathrm{TMZ}$ therapy was discontinued if clinical evidence for disease progression while on therapy was observed. No dose-limiting toxicities, as defined in the clinical protocol, were observed in any enrolled patients (see clinical protocol included in Supplemental Material; supplemental material available online with this article; doi:10.1172/JCI76739DS1).

Myelosuppression associated with single agent BCNU conditioning. With infusion of gene-modified CD $34^{+}$cells, hematopoietic suppression associated with BCNU conditioning was moderate: the lowest ANC observed was 30/microliter $(\mu \mathrm{l})$ (case patient 6) and the lowest PLT count was 28,000/ $\mu$ l (case patient 1). An ANC of less than $100 / \mu \mathrm{l}$ was observed in four of seven patients lasting no more than 4 days (range 1-4 days) and without fever. Mean hematopoietic recovery following BCNU, measured as both an ANC greater than $1,500 / \mu 1$ and PLT greater than $100,000 / \mu$ l, was 29 days after transplant (range 17-39 days) and no complications requiring intervention during the engraftment period were observed (Figure 2, B-H).

Myelosuppression associated with posttransplant $\mathrm{O}^{6} \mathrm{BG} / \mathrm{TMZ}$ chemotherapy. Myelosuppression following $\mathrm{O}^{6} \mathrm{BG} / \mathrm{TMZ}$ chemotherapy was observed in five of seven patients and occurred most frequently after the first, second, and third cycles, likely due to hematopoietic reconstitution by gene-modified short-term progenitors with limited repopulation capacity (Table 2). At 100 days after transplantation, the only incidence of significant chemotherapy-associated myelosuppression occurred in case patient 6 , who displayed little to no gene-modified (i.e., chemoprotected) cells in circulation at the time of chemotherapy (Figure 3). The unanticipated loss in long-term engraftment of gene-modified cells in 
A
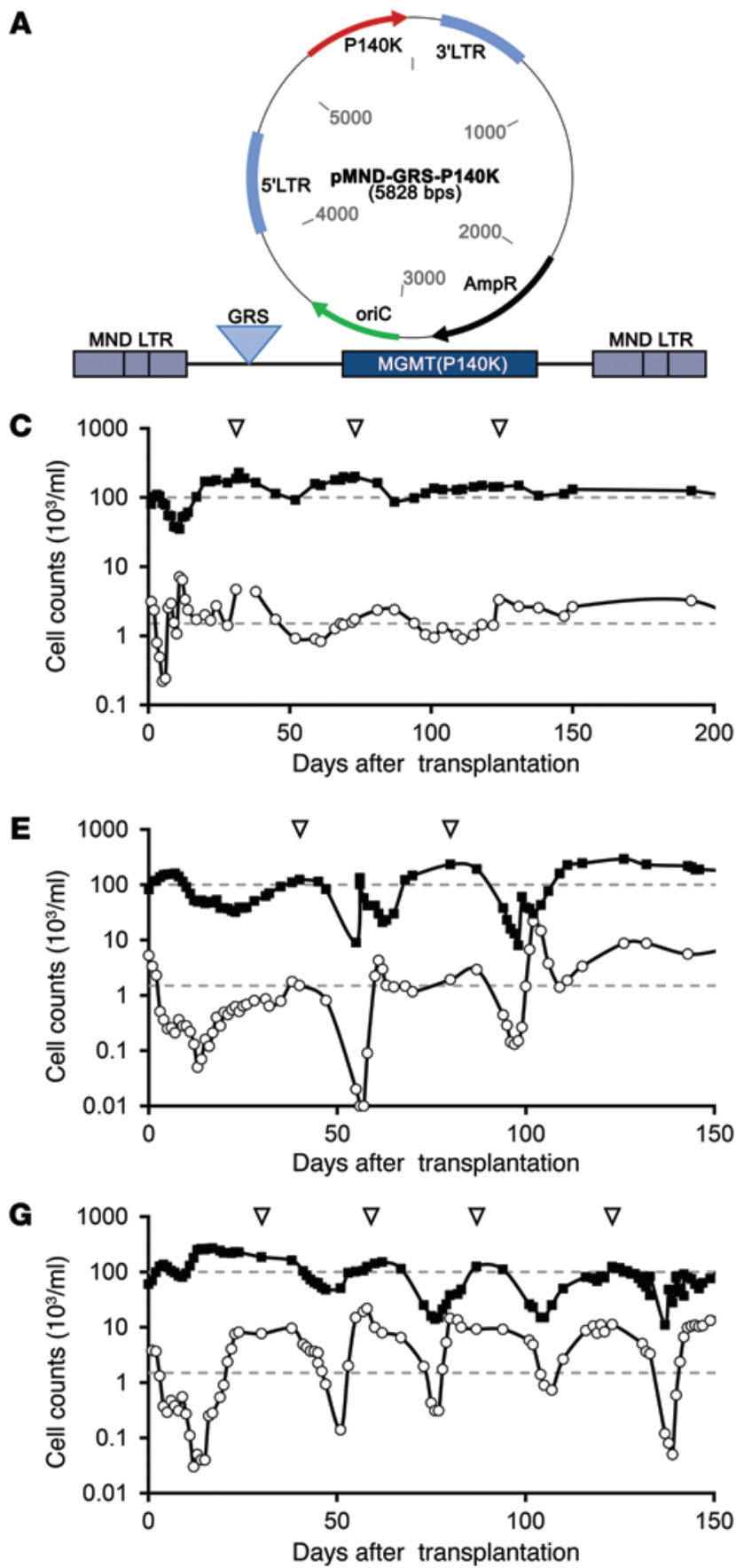

B

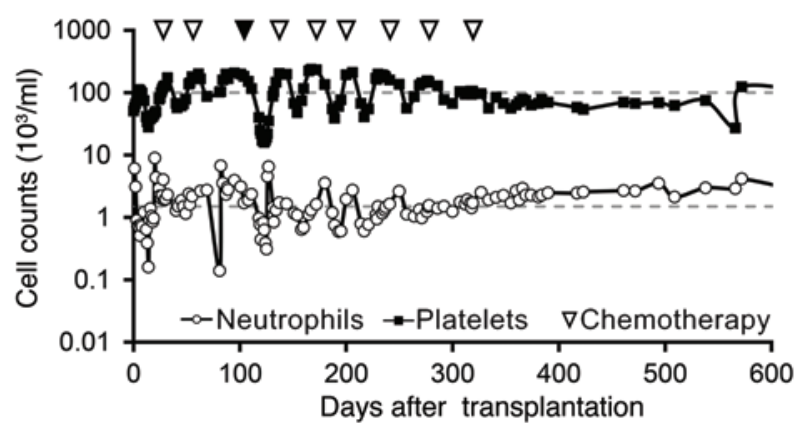

D

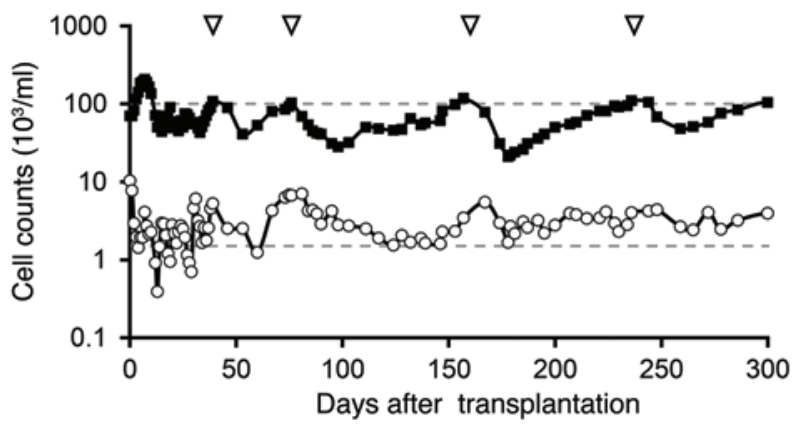

$\mathbf{F}$

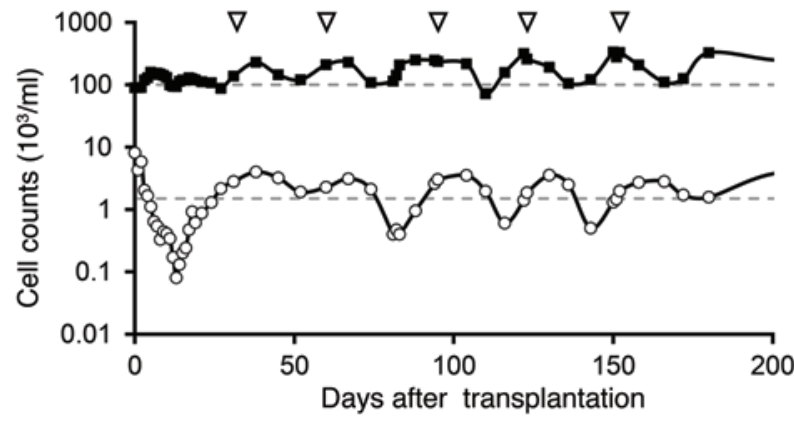

H

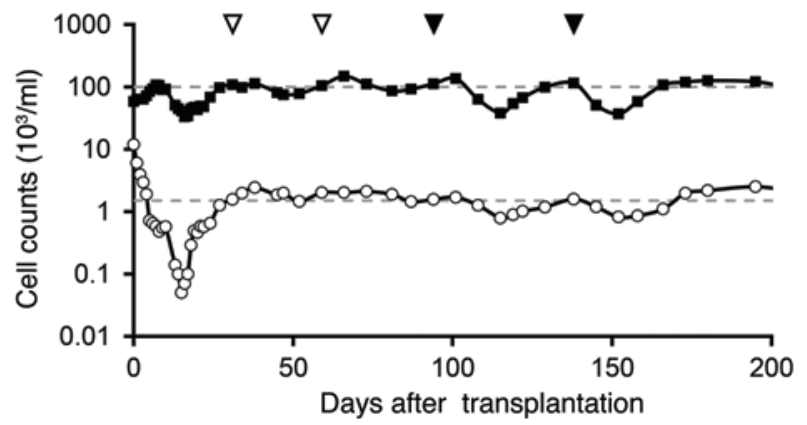

Figure 2. Chemoresistance of wbc in vivo after infusion of P140K gene-modified CD34+ cells and $\mathbf{O}^{6} \mathrm{BG} / \mathrm{TMZ}$ chemotherapy. (A) Schematic of the P140K-encoding gammaretrovirus vector used for transduction of autologous human CD34+ cells. (B-H) Blood cell counts for chemotherapy treatment per protocol (ANC and PLTs) for case patients 1 to 7 (B-H, respectively). Each graph shows ANC (white circles) and PLT counts (black squares) as measured from peripheral blood ( $y$ axis) over time ( $x$ axis) and chemotherapy with $0^{6} \mathrm{BG} / \mathrm{TMZ}$ (inverted triangles). Black inverted triangles in $\mathbf{B}$ (case patient 1 ) and $\mathbf{H}$ (case patient 7) represent dose-escalated chemotherapy cycles with TMZ dose of $590 \mathrm{mg} / \mathrm{m}^{2}$. Upper and lower dashed lines represent the threshold counts for each hematopoietic cell type required for chemotherapy retreatment in the study.

this patient resulted from a change in the lot formulation of commercial medium used during ex vivo culture of the cells for gene transfer, which is discussed in more detail below. Importantly, no significant myelosuppression was observed after the first 100 days following transplant in the patient with the longest follow-up (case patient 1 ), who displayed long-term engraftment of gene-modified cells and received an unprecedented 9 cycles of $\mathrm{O}^{6} \mathrm{BG} / \mathrm{TMZ}$ chemotherapy at or above the previously established MTD for TMZ (Supplemental Table 1).

Gene-modified cell responses. Transient increases in gene-modified granulocytes after chemotherapy corresponded with transient increases observed in total wbc populations and coincided with 


\section{Table 2. Chemotherapy-associated myelosuppression}

\begin{tabular}{|c|c|c|c|c|c|c|}
\hline GBM patient & Cycle no. & $\begin{array}{c}\text { Days ANC } \leq 500 / \mu \mathrm{l} \\
\text { (nadir) }\end{array}$ & $\begin{array}{c}\text { Days ANC } \leq 100 / \mu \mathrm{l} \\
\text { (nadir) }\end{array}$ & $\begin{array}{c}\text { Days PLTs } \leq 25,000 / \mu \mathrm{l} \\
\text { (nadir) }\end{array}$ & $\begin{array}{c}\text { Days PLTs } \leq 10,000 / \mu \mathrm{l} \\
\text { (Nadir) }\end{array}$ & $\begin{array}{l}\text { Grade } 4 \text { nonhematologic } \\
\text { toxicity observed }\end{array}$ \\
\hline \multirow[t]{2}{*}{ Case 1} & 2 & $1(140)^{A}$ & - & - & - & - \\
\hline & $3^{\mathrm{B}}$ & $3(310)$ & - & $7(16,000)$ & - & - \\
\hline Case 3 & 3 & - & - & $3(21,000)$ & - & - \\
\hline Case 4 & 1 & 5 & 5 (None) ${ }^{A}$ & 3 & $1(9,000)^{A}$ & - \\
\hline Case 5 & 2 & $3(400)$ & - & - & - & - \\
\hline \multirow[t]{3}{*}{ Case 6} & 1 & $1(140)$ & - & - & - & - \\
\hline & 2 & $3(310)$ & - & $4(14,000)$ & - & - \\
\hline & $4^{c}$ & 3 & $2(50)$ & $1(11,000)$ & - & - \\
\hline
\end{tabular}

${ }^{A}$ Required intervention: for neutropenia, G-CSF administration; for thrombocytopenia, transfusion. ${ }^{\mathrm{B} T M Z}$ dose-escalated cycle. ${ }^{\mathrm{C}} \mathrm{Patient}$ displayed low to undetectable gene-modified cells in peripheral blood at the time of chemotherapy.

nadir neutropenia and thrombocytopenia after each cycle, demonstrating protection of gene-modified peripheral blood cells (compare Figure 2 with Figure 3). In three patients (case patients 4-6), a loss of detectable gene-modified cells in the peripheral blood was observed between 40 and 100 days after transplant (Figure 3, D-F). Further review of manufacturing records revealed a correlation between the commercial lot of HSC culture medium used during ex vivo gene transfer into $\mathrm{CD} 34^{+}$hematopoietic cells from these patients compared with the first three patients, all of whom demonstrated persistent gene-modified cell engraftment. There were no observable significant differences in cell fitness at the time of transplant based on cell dose and viability, efficiency of gene transfer, or colony-forming capacity among these 6 patients (Supplemental Table 2); thus, the observed differences in long-term engraftment in vivo were unanticipated. Through communication with the manufacturer, the difference in media lot performance was attributed to bovine serum albumin used during formulation. We therefore switched to a xenogen-free version of HSC supportive medium for the subsequent gene modification of autologous $\mathrm{CD} 34^{+}$cells from case patient 7 . Following gene transfer, we observed no significant difference in cell dose and viability, efficiency of gene transfer, or colony-forming capacity compared with the previous 6 patients (Supplemental Table 2), but we did observe robust, longterm engraftment of gene-modified cells in this patient throughout chemotherapy (Figure 3G). Interestingly, in the four patients with durable gene marking throughout chemotherapy (patients 1, 2, 3, and 7), we observed a gradual decline in circulating gene-modified peripheral blood cell levels immediately following discontinuation of chemotherapy, suggesting a potential disadvantage for genemodified stem cells in the absence of chemoselective pressure.

Patient responses. All seven case patients were evaluable for response throughout treatment. We observed one partial response (PR) per Revised Assessment in Neuro-Oncology (RANO) (11) criteria while on study treatment (case patient 1 ). While this response was durable throughout therapy, declining levels of circulating genemodified cells observed in this patient between cycles seven and nine warranted discontinuation of $\mathrm{O}^{6} \mathrm{BG} / \mathrm{TMZ}$ treatment. This patient received no further treatment for GBM and more recently demonstrated complete response (CR) (Figure 4). Six out of seven patients demonstrated progressive disease while on $\mathrm{O}^{6} \mathrm{BG} / \mathrm{TMZ}$ therapy with a median progression-free survival (PFS) from diagnosis of 9 months (Table 1). While on therapy, five of these six patients demonstrated a best response of stable disease by RANO criteria and one patient (case patient 6) demonstrated progressive disease. Median OS was 20 months for the seven patients treated to date, and we observed $100 \%$ survival of enrolled patients at one year from diagnosis. Six of the seven patients are evaluable for two-year survival from diagnosis at the time of this writing. We observed three of seven patients alive at two years, a significant improvement compared with historical MGMThi patients receiving RT alone as upfront therapy followed by adjuvant TMZ ( 1 of $54 ; P=0.02)(2)$. Moreover, all seven patients surpassed the median survival for patients in the same RPA class: reported median OS is 17.1 months, 11.2 months, and 7.5 months for RPA classes III, IV, and V, respectively (10).

Biomathematical modeling to evaluate $O^{6} B G$ benefit. To determine potential therapeutic gain achieved with concomitant $\mathrm{O}^{6} \mathrm{BG}$ administration during the adjuvant phase of TMZ therapy, we applied patient-specific biomathematical modeling of tumor growth (12). We examined two pretreatment MRI scans (typically at presentation and immediately prior to surgical resection), preferably on the same MRI machine to accurately compute model kinetics. We then predicted the growth of each patient's tumor, if left untreated, in terms of millimeters ( $\mathrm{mm})$ of radial tumor growth per day (Figure 4). The resulting patient-specific "untreated virtual control" (UVC) provided the baseline against which treatment effect was measured (Figure 4, plot of untreated radial tumor growth versus time) $(12,13)$. Treatment effect was described in two ways: first, as the $x$ axis deflection from the UVC, days gained (DG, defined as number of days the treatment delayed tumor progression), and second, as the $y$ axis deflection from the UVC, radial treatment response (RTR, defined as the radius of tumor growth prevented). Of the seven patients treated, four had the required pretreatment imaging necessary to reliably model untreated tumor growth (case patients 1-4). 

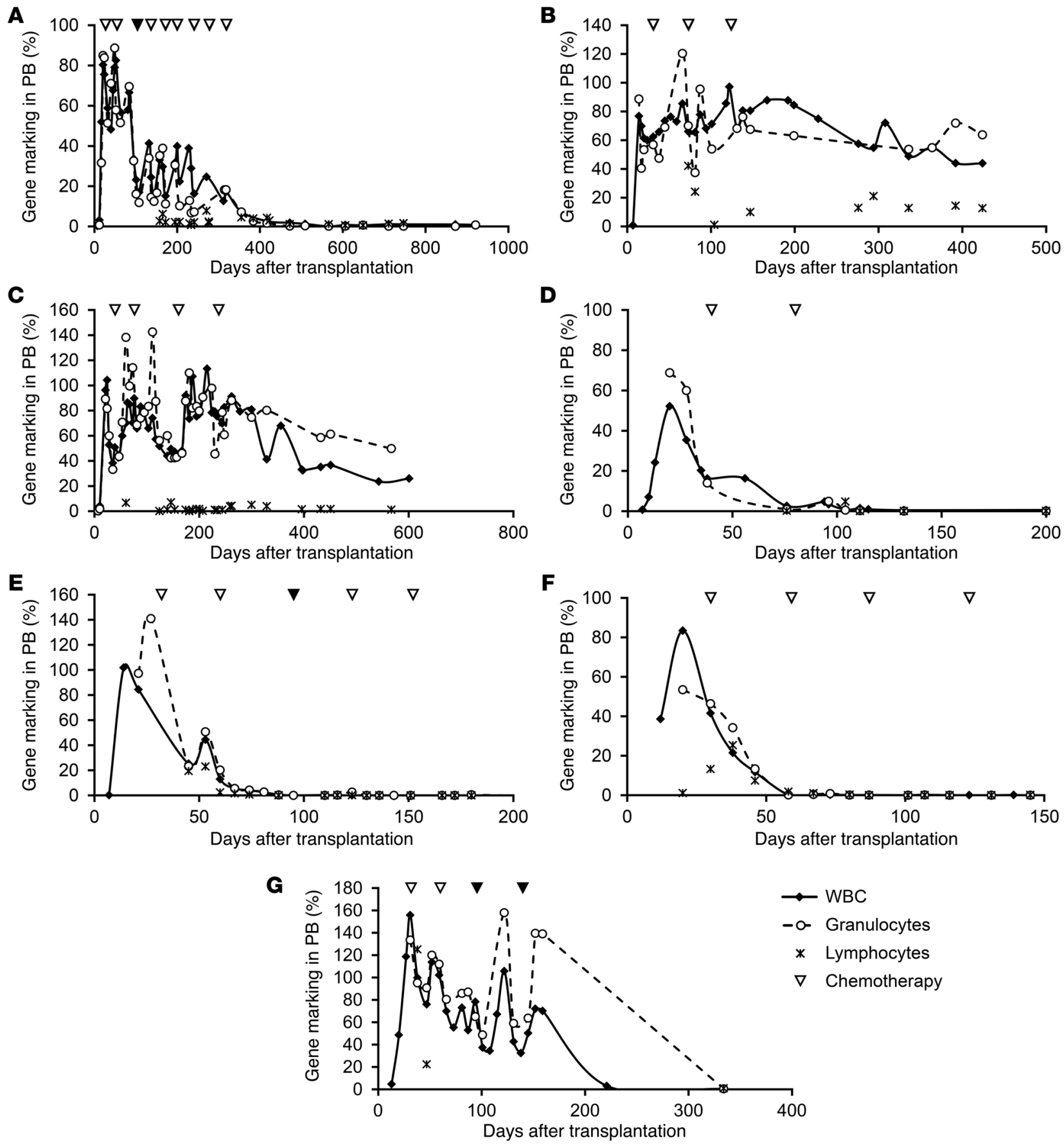

Figure 3. Persistence of P140K gene-modified cells in vivo. Percentage of gene-modified wbc within circulating peripheral blood of patients 1 to 7 are represented in A-G, respectively. Each graph represents the percentage of circulating bulk wbc (black diamonds), granulocytes (white circles), and lymphocytes (asterisks), with integrated transgene-containing provirus by PCR assay ( $y$ axis) over time ( $x$ axis). Chemotherapy (inverted triangles) is shown as well.

We observed a robust deflection of tumor growth from the patient-specific UVC at each time point of response evaluation analyzed for all four patients, (Supplemental Figure 1 and Supplemental Figure 2). For the three patients for whom multiple response evaluations were available (case patients 1-3), we observed increased deflection from the UVC over time and with additional TMZ administration (Table 3).

We then assessed whether this observed deflection in tumor growth with combined TMZ and $\mathrm{O}^{6} \mathrm{BG}$ provided a substantial treat- ment benefit over standard therapy (TMZ alone) by comparing treatment responses observed in study (case) patients with those of five control patients who received standard therapy and were treated at our institution (Table 3). We matched patients for comparison by similarity of model-determined disease kinetics (net invasion rate, $D$, and net proliferation rate, $\rho$ ) and similar prognostic clinical characteristics, including diagnosis of GBM, extent of surgical resection (EOR), RPA class, age, and Karnofsky Performance Status (KPS) (14) at time of diagnosis. MGMT promoter methylation 

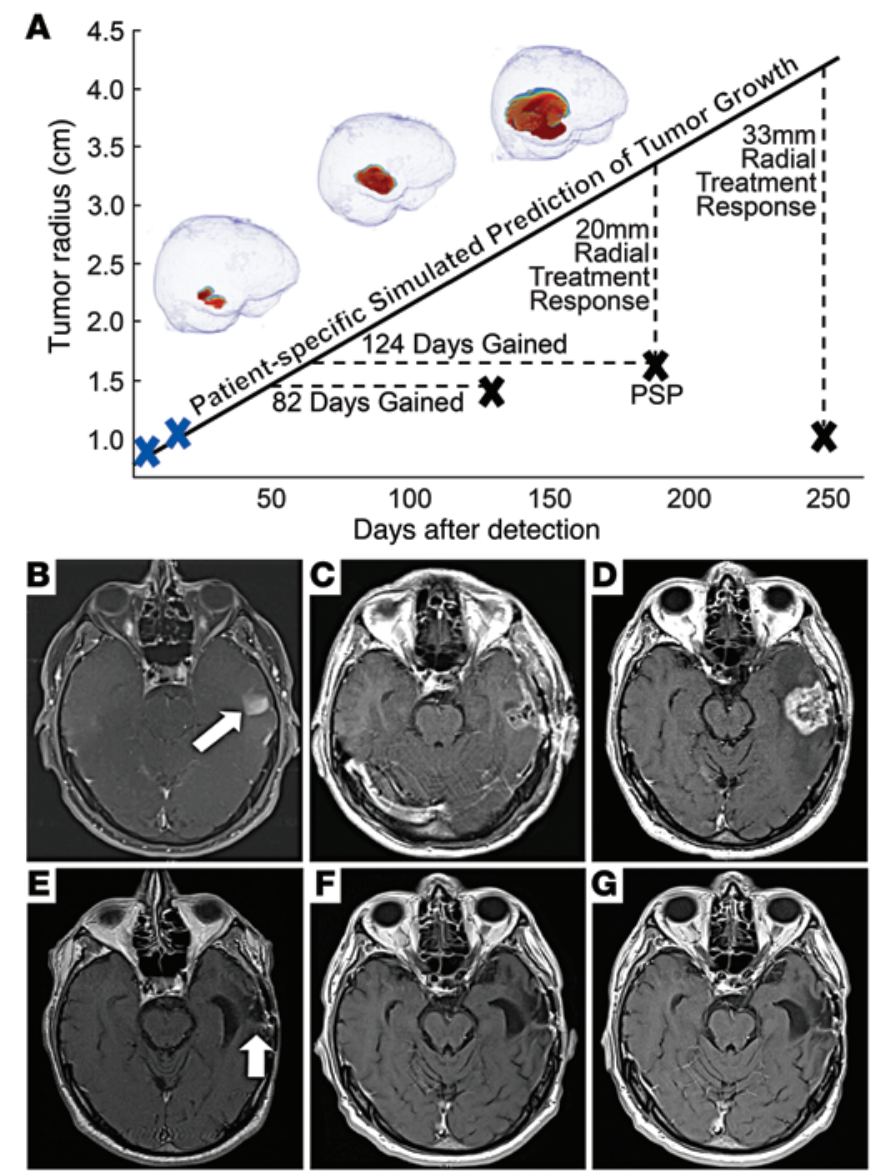

status for control patients was also determined, since this variable has been prognostic for survival benefit achieved with standard care TMZ regimens (Supplemental Table 3). For patient 1, two suitable controls were identified (control 1A and 1B). Velocity of tumor growth was greater in case patients $(\mathrm{mm} / \mathrm{d}$ : range $0.11-0.66$, mean $0.34)$ compared with controls $(\mathrm{mm} / \mathrm{d}$ : range $0.035-0.26$, mean $0.13)$, but was not significantly different in distribution $(P>0.10)$. All case patients demonstrated greater average DG and prevention of radial tumor growth per mg of TMZ administered compared with matched controls, which was sustained for the duration of the treat-
Figure 4. Patient-specific biomathematical modeling of tumor growth and treatment response. (A) UVC for predicted tumor growth if left untreated for case patient 1. Two initial pretreatment MRI scans (blue $x$ 's) were used to determine the predicted tumor growth (solid line) if left untreated. The first MRI was obtained on the day of clinical presentation, and the second MRI was obtained as part of neuronavigational guidance protocol on the day of resection (day 10). Tumor radius ( $y$ axis) is determined from follow-up posttreatment MRIs (black x's) and plotted against time since the first MRI in days ( $x$ axis). The treated tumor radius is assessed relative to the projected untreated growth to determine the response measured as DC or RTR. Note: Increase in enhancement observed by MRI at 182 days ( $\mathbf{D}$, below) was determined to be consistent with pseudoprogression (PSP) by second surgical resection. (B-G) Observed clinical response in case patient 1 . Contrast-enhanced MRI scans obtained, in chronological order, at initial diagnosis (B, second gray $x$ denoted in $\mathbf{A}$ ), after RT and transplant (before $0^{6} \mathrm{BC} / \mathrm{TMZ}$ chemotherapy) (C), following cycle II of $0^{6} \mathrm{BG} / \mathrm{TMZ}$ chemotherapy (interval increase representative of pseudoprogression corresponding to second black x in A) (D), following second surgical resection, which indicated pseudoprogression (for pathology, see Supplemental Figure 3) (E), following cycle 9 of $0^{6} \mathrm{BC} / \mathrm{TMZ}$ chemotherapy (F), and at 2 years since diagnosis (11 months after therapy discontinuation) (G).

\section{Table 3. Treatment benefit values for each patient analyzed by biomathematical modeling}

\begin{tabular}{|c|c|c|c|c|c|c|c|c|}
\hline GBM patient & $\begin{array}{l}\text { Total no. of evaluable } \\
\text { time points after RT }\end{array}$ & $\begin{array}{l}\text { Total TMZ dose } \\
\text { administered (mg) }\end{array}$ & $\begin{array}{l}\text { DGmg } \\
\text { TMZ }^{\text {intital }}\end{array}$ & $\begin{array}{l}\mathrm{DC} / \mathrm{mg} \\
\mathrm{TMZ}^{\text {final }}\end{array}$ & $\begin{array}{c}\text { Mean DG/mg } \\
\text { TMZ }\end{array}$ & $\begin{array}{c}\text { Radial tumor } \\
\text { response/mg TMZ }{ }^{\text {initial }}\end{array}$ & $\begin{array}{l}\text { Radial tumor } \\
\text { response/mg TMZ final }\end{array}$ & $\begin{array}{l}\text { Mean radial tumor } \\
\text { response/mg TMZ }\end{array}$ \\
\hline Control 1A & 2 & 4900 & $2.8 \times 10^{-2}$ & $1.0 \times 10^{-4}$ & $1.4 \times 10^{-2}$ & $1.3 \times 10^{-3}$ & $1.4 \times 10^{-3}$ & $1.4 \times 10^{-3}$ \\
\hline Case 2 & 2 & 2475 & $4.3 \times 10^{-2}$ & $5.2 \times 10^{-2}$ & $4.8 \times 10^{-2}$ & $1.3 \times 10^{-2}$ & $1.2 \times 10^{-2}$ & $4.1 \times 10^{-2}$ \\
\hline Control 2 & 4 & 4985 & $4.2 \times 10^{-3}$ & $-8.2 \times 10^{-3}$ & $2.4 \times 10^{-3}$ & $1.0 \times 10^{-3}$ & $2.0 \times 10^{-4}$ & $4.7 \times 10^{-3}$ \\
\hline Case 3 & 3 & 4180 & $3.4 \times 10^{-2}$ & $5.2 \times 10^{-2}$ & $4.5 \times 10^{-2}$ & $4.5 \times 10^{-2}$ & $3.8 \times 10^{-2}$ & $1.2 \times 10^{-2}$ \\
\hline Case 4 & 1 & 1560 & $5.2 \times 10^{-2}$ & ND & $5.2 \times 10^{-2 A}$ & $9.0 \times 10^{-2}$ & ND & $9.0 \times 10^{-2 A}$ \\
\hline Control 4 & 6 & 21455 & $2.0 \times 10^{-2}$ & $1.5 \times 10^{-2}$ & $1.7 \times 10^{-2}$ & $2.7 \times 10^{-3}$ & $2.2 \times 10^{-3}$ & $2.4 \times 10^{-3}$ \\
\hline
\end{tabular}

$\mathrm{ND}$, unable to determine since only one posttreatment response evaluation was available. ${ }^{\mathrm{A}}$ Only one posttreatment value was included in the mean calculation.

ment course (Figure 5 and Table 3). Case patient 4 displayed a single value for both parameters measured that was greater than that observed over all time points for the corresponding matched control patient 4, despite receiving fewer TMZ chemotherapy cycles.

\section{Discussion}

Here, we show that gene therapy to protect HSCs and progenitor cells from the toxic effect of $\mathrm{O}^{6} \mathrm{BG} / \mathrm{TMZ}$ chemotherapy is feasible and allows for the administration of significantly more chemotherapy cycles in patients with newly diagnosed, poor-prognosis GBM 

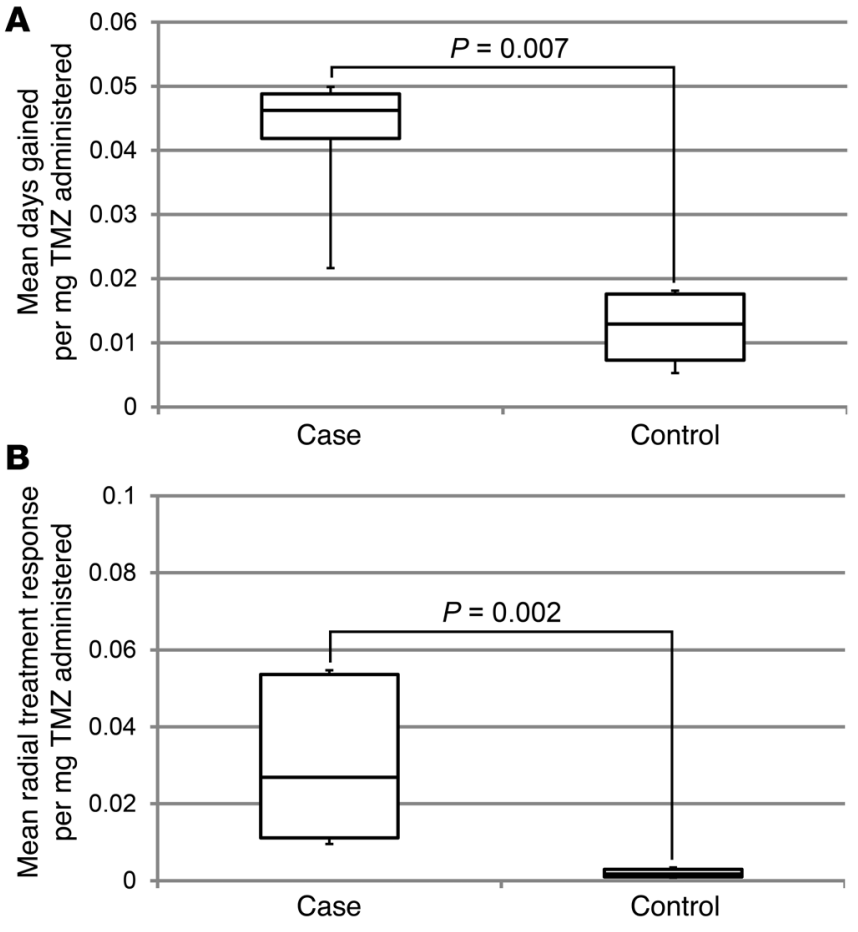

Figure 5. Biomathematical modeling reveals greater response to treatment as a function of TMZ administered in study case patients versus matched controls. (A) Box and whisker plots illustrating the significant difference in mean DG per milligram of TMZ received observed between study patients (case) and matched patients receiving standard care regimens (control). (B) Box and whisker plots illustrating the significant difference in mean RTR per milligram of TMZ received observed between study patients and matched patients receiving standard care regimens. For each plot, box bounds represent the first quartile (lower bound) and third quartile (upper bound). Lines within the box represent the median. Whiskers represent the difference from the minimum value observed in the data set to the first quartile (lower whisker) and the difference from the third quartile to the maximum value observed (upper whisker).

than previously described in patients without the benefit of chemoprotected blood cells (6). We observed no significant hematologic toxicity from chemotherapy following the first 100 days after transplant of chemoprotected, gene-modified cells, permitting up to nine cycles of $\mathrm{O}^{6} \mathrm{BG} / \mathrm{TMZ}$ chemotherapy. Importantly, we demonstrate that the ability to give significantly more $\mathrm{O}^{6} \mathrm{BG} / \mathrm{TMZ}$ cycles was associated with a significant improvement in overall outcome. Specifically, we observed a significant improvement in two-year survival in patients treated with gene therapy compared with historical patients receiving $\mathrm{TMZ}$ without the benefit of $\mathrm{O}^{6} \mathrm{BG}$ (2).

Given the high frequency of GBM patients with unmethylated MGMT promoters in tumor cells ( $\sim 50 \%$ of both young and elderly patients), there is a substantial need to develop treatment strategies that improve the standard care approach $(3,15)$. In patients classified as Radiation Therapy Oncology Group (RTOG)/RPA class IV, median OS is 11.2 months, and 1-year survival rates are $46 \%$ (10). Six of the seven patients treated in this category were classified as RPA class IV (case patients 1, 2, 3, 4, 6, and 7), and all surpassed this median expected survival (range $14-57^{+}$months). In the single RPA class III patient enrolled and treated (case patient 5), we still observed an improvement in survival over the expected: RPA class
III median survival is 17.1 months, with a 70\% 1-year survival rate, while patient 5 survived 28 months. For all categories, MGMT ${ }^{\text {hi }}$ status is considered a poor prognostic indicator for OS; thus, the $100 \%$ survival rate at one year and the $33 \%$ survival rate at two years from diagnosis observed in our study are highly significant. In addition, case patients 1,3 , and 5 have demonstrated significantly extended OS, surpassing even median survival for MGMT ${ }^{\text {lo }}$ tumor status patients receiving standard therapy (21.7 months) (2). Though these phase I study results are encouraging, we cautiously interpret clinical benefit compared with historical response, as true clinical benefit over standard therapy can only be detected following a phase III, randomized, double-blinded clinical trial.

Most notably, addition of $\mathrm{O}^{6} \mathrm{BG}$ to the adjuvant TMZ regimen permitted the observed improvement in OS to be achieved at far lower cumulative TMZ doses than typically received by GBM patients in standard care regimens. Case patient 1 , who displayed the most robust response, received 9,480 total $\mathrm{mg}$ of TMZ during nine cycles of chemotherapy treatment, whereas his corresponding institutional matched controls received 4,900 and 9,650 $\mathrm{mg}$ $\mathrm{TMZ}$, respectively, in only three cycles of treatment.

To characterize the treatment benefits achieved with $\mathrm{O}^{6} \mathrm{BG}$ added to the TMZ regimen, we applied a precision medicine, biomathematical model to compare the treatment strategy employed in study patients with standard care regimens in patients treated at our institution (12, 13, 16-20). We show that model-predicted, untreated tumor growth was deflected by a greater degree in study patients compared with matched control patients, at lower cumulative TMZ doses when administered with $\mathrm{O}^{6} \mathrm{BG}$.

There are several assumptions implicit in using modeling approaches, such as the approach applied here. In this case, we assumed that patient-individualized predictions of tumor growth, if left untreated, were consistent with a fixed/intrinsic tumor kinetic. With treatment, those rates can change, but the UVC is informed by this baseline expectation of consistency. We have shown that this kinetic is consistent over as much as 15 years of imaging followup without treatment in low-grade gliomas (21) and for over 1 year in rare, untreated GBM tumors (19). However, we were only able to predict untreated tumor growth in four of seven treated patients, owing to inconsistencies in pretreatment imaging. Addressing this will likely require improvements in both pretreatment imaging standards and biomathematical modeling. Importantly, for the patients evaluated, tumor growth velocity was similar between treatment groups and, thus, does not explain the differences in treatment gains observed. For the study patients evaluated, increasing response values per $\mathrm{mg}$ of TMZ suggest a potentiating efficacy with successive rounds of TMZ. This supports our original hypothesis that the ability to deliver multiple cycles of TMZ in combination with $\mathrm{O}^{6} \mathrm{BG}$ results in improved tumor response and overall outcome in poor-prognosis GBM patients.

In a prior phase II clinical trial of $\mathrm{O}^{6} \mathrm{BG}$ and $\mathrm{TMZ}$ chemotherapy in recurrent GBM patients with TMZ-resistant tumors, a significant improvement in OS and PFS was not observed, primarily owing to a dose reduction of TMZ required $(50 \%)$ to avoid hematologic toxicity (6). While our study treated newly diagnosed GBM patients with TMZ-resistant tumors based on MGMT promoter methylation status, our findings suggest that the ability to administer $\mathrm{O}^{6} \mathrm{BG} / \mathrm{TMZ}$ at or above the previously described MTD 
for TMZ by chemoprotection of the hematopoietic system can improve response. Moreover, our data suggest that between six and nine cycles of this chemotherapy regimen may be all that is needed to elicit a prolonged therapeutic benefit.

The observed decline in gene-modified cells following therapy discontinuation in patients with durable gene marking throughout treatment could indicate that constitutive MGMT expression in blood cells may not be ideal. We envision that this limitation could be overcome by use of a less active or regulated (chemotherapy inducible) promoter to drive MGMT expression in gene-modified cells. Furthermore, the loss in long-term repopulating gene-modified cells resulting from lot-to-lot variation in commercial cell culture medium used during gene transfer highlights the need to reduce xenogen exposure during gene transfer, as well as to develop more potent tests for HSC fitness ex vivo. Importantly, given the improved therapeutic benefit and OS observed in all patients treated in the current study, these findings also suggest that permanent gene modification may not be required to elicit an improvement in outcome. Based on our results, six to nine cycles of $\mathrm{O}^{6} \mathrm{BG} / \mathrm{TMZ}$ chemotherapy can substantially improve outcome; therefore, prolonged gene modification of peripheral blood cells is not necessary.

The results of this study indicate that gene modification with the $\mathrm{O}^{6} \mathrm{BG}$-resistant P14OK mutant MGMT can chemoprotect the blood and bone marrow from myelosuppression associated with combined $\mathrm{O}^{6} \mathrm{BG}$ and TMZ chemotherapy. Moreover, addition of $\mathrm{O}^{6} \mathrm{BG}$ to TMZ in the post-RT milieu can provide significant treatment gains per mg of TMZ dose compared with TMZ alone. This consistent pattern of superior treatment response in study patients provides strong evidence for increased therapeutic benefit of the study protocol over standard of care regimens for MGMThi GBM patients, supporting continued investigation of this approach.

In conclusion, our results show that $\mathrm{P} 140 \mathrm{~K}$-modified HSCs and progenitor cells, in combination with dose-intense TMZ and $\mathrm{O}^{6} \mathrm{BG}$, is a clinically feasible alternative to standard therapy for poor prognosis, MGMT ${ }^{\text {hi }}$ GBM patients. Importantly this also has implications for other MGMT-overexpressing brain tumors or even other types of tumors for which alkylating agent chemotherapy could be used for treatment.

\section{Methods}

Study design. The clinical trial (National Clinical Trials Registry, NCT00669669) was designed to evaluate safety and feasibility of autologous $\mathrm{P} 140$ K gene-modified $\mathrm{CD} 34^{+}$hematopoietic cell infusion in newly diagnosed MGMT ${ }^{\text {hi }}$ GBM. Study patients were treated at the UW Medical Center or at the Seattle Cancer Care Alliance (SCCA), as previously described (9). Institutional review board approvals were secured for both clinical trial conduct and biomathematical modeling analyses. The study was conducted in accordance with the protocol (see clinical protocol in the Supplemental Material). No commercial sponsor was involved in the study. $\mathrm{O}^{6} \mathrm{BG}$ was provided for this study by the Cancer Therapy Evaluation Program of the National Cancer Institute (NCI). Toxicity was assessed according to the NCI Common Toxicity Criteria version CTCAEv3.

Study procedures for enrolled patient. Study patients underwent fractionated, involved field, external beam radiotherapy without concomitant TMZ. RT was administered daily in fractions of $2 \mathrm{~Gy}$, given five days per week, for six weeks, for a total of $60 \mathrm{~Gy}$. Concomitant TMZ was eliminated during RT per study protocol since (a) the desired autologous HSCs used for subsequent gene transfer could be compromised by this treatment and (b) development of increased tumor cell resistance to TMZ was likely given MGMThi tumor status. Patients were then mobilized with G-CSF $(16 \mu \mathrm{g} / \mathrm{kg} / \mathrm{d})$ alone for five days with mobilized CD34 cell counts on days three to five. Patients demonstrating a peripheral blood $\mathrm{CD} 34^{+}$cell count of $5 / \mu$ l or more on day 3 were increased to $32 \mu \mathrm{g} / \mathrm{kg} / \mathrm{d}$ G-CSF fractionated into two $16 \mu \mathrm{g} / \mathrm{kg}$ doses given approximately 12 hours apart in combination with plerixafor (AMD3100; $240 \mu \mathrm{g} / \mathrm{kg}$ administered 1 hour after the evening dose of G-CSF). On days four and five of mobilization, patients underwent two successive, large-volume leukapheresis procedures to collect CD34 ${ }^{+}$ hematopoietic cells. One to two days later, patients received a single infusion of $600 \mathrm{mg} / \mathrm{m}^{2} \mathrm{BCNU}$ as both chemotherapy for residual disease and conditioning to facilitate engraftment of gene-modified cells. Gene-modified cells infusions occurred between 24 and 48 hours following administration of BCNU.

The vector used in these studies, MND-GRS-P140K, is a gibbonape leukemia virus-pseudotyped (GALV-pseudotyped) gammaretroviral vector encoding the P14OK cDNA, which was tested for safety and functionality preclinically, as previously reported (8). CD34 ${ }^{+}$cells were selected and transduced as previously described (9). Quantitative real-time PCR (TaqMan PCR) was performed to detect provirus sequences in peripheral blood and bone marrow cells, and standard PCR was used to detect provirus sequences in colony-forming CD $34^{+}$ hematopoietic cells as previously described $(8,22)$.

Biomathematical modeling of treatment benefit. Biomathematical modeling studies were performed in the laboratory of Kristin Swanson (Northwestern University, Evanston, Illinois, USA). The model used is a biologically based, patient-specific mathematical model for quantifying the growth of gliomas through the estimation of patientcalibrated net rates of proliferation $(\rho)$ and invasion $(D)$ of tumor cells, referred to as the proliferation-invasion (PI) model (12, 13, 16-20, 22-25), which has previously been shown to generate accurate predictions of GBM growth in untreated $(19,21,24)$ and treated contexts (20). The model was applied to predict the growth of the tumor, if left untreated, holding rate parameters constant. This UVC provided a baseline against which treatment effect was measured (Figure 4 and refs. 12, 13).

Using the model, patient-tuned parameters $\rho$ and $D$ define the profile of the leading edge of the tumor and the speed (velocity V) at which it would be expected to grow untreated $(16,18,19,23,24)$ or treated (20). For these analyses, values of $\rho$ and $D$ were calculated for purposes of matching tumor growth characteristics between case and control patients, and velocity was used to simulate untreated tumor growth (13). As previously reported (16), the parameters were tuned by fitting two serial, pretreatment MRI scans (one obtained at the time of symptom presentation and the other at the time of surgery) obtained on 1.5T and 3.0T Philips machines with routine clinical MR pulse sequences; slice thickness in the axial plane varied from $1 \mathrm{~mm}$ to $6 \mathrm{~mm}$. Tumor volumes observed on pretreatment gadoliniumenhanced MRIs were computed using a semiautomated intensity threshold-based segmentation software developed by MATLAB (version 7.13.0.564 [R2011b]; The MathWorks, Inc.). Tumor volumes were calculated from contrast-enhancing regions on T1-weighted gadolinium-enhanced (T1GD) and T2-weighted MRIs in the axial view, as 
measured by at least two observers. The arithmetic mean of these volumetric measurements was converted into a spherically equivalent radius from which the velocity of radial expansion $(V, \mathrm{~mm} / \mathrm{d})$ was computed as the difference in tumor radius of the two pretreatment scans (Time2 - Time1) divided by the number of days between the MRI scans. All tumor velocity measurements were computed using the T1GD volume data except for study case patient 4, in which the T2 MRI volume data was used to calculate a growth velocity. Treatmentresponse values for study case patient 4 were still computed with T1GD MRI image measurements.

Calculation of therapeutic benefit by biomathematical modeling. Cumulative DG per mg of TMZ administered was computed at each MRI time point during and following treatment. The baseline MRI time point $\left(t_{1}\right)$ occurred before the first cycle of known TMZ dosing. Medical record data was used to obtain the total amount of TMZ received in mg between the baseline MRI scan and the subsequent posttreatment follow-up MRI scan at time point, $t$. The number of cumulative days of treatment between the baseline MRI and subsequent images was computed as $\left(\Delta t=t_{i}-t_{1}\right)$. Change $(+/-)$ in tumor radius $(\Delta R)$ was determined as $\left(R_{i}-R_{1}\right)$, and the velocity of untreated radial expansion $(V, \mathrm{~mm} / \mathrm{d})$ was determined as described above. Each cumulative DG interval was calculated as the change in tumor radius divided by the velocity, $V$, subtracted from the time interval between MRI scans. The following formula was used to calculate the cumulative DG per cumulative $\mathrm{mg}$ of TMZ administered in the time interval $\Delta t$ :

$$
\frac{\text { cumulative } \mathrm{DG}}{\text { total } \mathrm{mg} \text { of } \mathrm{TMZ}}=\frac{\left(\Delta t-\frac{\Delta R}{\mathrm{~V}}\right)}{\text { total } \mathrm{mg} \text { of } \mathrm{TMZ}}
$$

(Equation 1)

We also quantified to what degree treatment deflected the predicted untreated tumor growth, defined as RTR (Figure 4 and Table 3). This difference is calculated as the UVC predicted tumor radius (product of velocity and time plus the initial tumor volume) less the actual tumor radius observed on MRI imaging at a given response evaluation time point.

Identification of matched control patients. We chose patient-specific controls with similar disease kinetics (net invasion rate, $D$, and net proliferation rate, $\rho$ ) and similar prognostic clinical characteristics, including diagnosis of GBM, EOR, RTOG/RPA class (26), age, and KPS at time of diagnosis. MGMT promoter methylation status for control patients was also determined, since this variable has been prognostic for survival benefit achieved with standard care TMZ regimens (see Table 1).

For study case patient 1, two suitable controls were identified: control 1A had received RT only as upfront therapy, as did case patient 1, followed by adjuvant TMZ per standard-of-care (SOC) dosing, whereas control $1 \mathrm{~B}$ received concurrent $\mathrm{RT}$ plus TMZ as upfront therapy, followed by adjuvant TMZ following a standard dosing schedule, but was more closely matched with case patient 1 by parameters of velocity of tumor growth, age, and KPS (Table 1). Case patient 2 and matched control patient 2 underwent gross total resection (GTR) of the primary tumor and were classified as RTOG/RPA class IV. Control patient 2 received SOC concurrent RT plus TMZ as upfront therapy, followed by adjuvant TMZ following a standard dosing schedule in conjunction with Rindopepimut vaccine. Case patient 3 and control patient 3 had subtotal resections and were classified as RTOG/RPA class IV. Control patient 3 received SOC concurrent RT plus TMZ as upfront therapy followed by adjuvant TMZ following a standard dosing schedule. Case patient 4 and control patient 4 both underwent GTR and were classified as RTOG/RPA class V. Control patient 4 received SOC concurrent RT plus TMZ as upfront therapy followed by adjuvant TMZ following a standard dosing schedule.

Statistics. Fisher's exact test was used to determine two-year survival benefit between case patients and historical controls (2). Student's $t$ test (2 tailed, paired) was used to determine significance of differences observed between case and institutional control patient values of OS, initial tumor growth velocity, DG per mg of TMZ administered and RTR per mg of TMZ administered. For case patient 1, two matched institutional controls (1A and $1 \mathrm{~B})$ were suitable for comparison. For statistical analyses of OS, DG, and RTR, the respective values for these two controls were averaged to produce a single value for comparison. A $P$ value of less than 0.05 was considered significant.

Study approval. All research performed in this study was approved by the Institutional Review Boards of the Fred Hutchinson Cancer Research Center (gene therapy protocol; principal investigator [P.I.], Hans-Peter Kiem), UW, and Northwestern University (tumor modeling protocols; P.I. Kristin R. Swanson). Informed consent for all patients was obtained prior to treatment, and all research was conducted within the principles expressed by the Declaration of Helsinki.

\section{Acknowledgments}

We thank Kenneth Cornetta and the Indiana University Virus Production Facility for production of the clinical grade virus used in this study. Devikha Chandrasekaran, Christina Ironside, Nathaniel Williams, and Garrick Horn are acknowledged for technical assistance in conduct of this work. We thank the Gene and Cell Therapy Laboratory of UW, the Cell Processing Facility of the Fred Hutchinson Cancer Research Center, and the Cellular Therapy Laboratory of the SCCA for support during postcollection processing and transduction. We also thank all clinical service teams involved in the conduct of this study including the Neuro-Oncology and Infusion Service teams of the UW Medical Center and SCCA, the Neurological Surgery and Radiation Oncology teams of the UW Medical Center and the Gold Autologous Transplant Team of the SCCA. We appreciate Grace Choi's assistance in the collection of clinical data and preparation of the manuscript. The authors are grateful for research funding from the NIH (grants R01CA114218, R01AI080326, R01HL098489, P30DK056465, and R01HL074162 to H.-P. Kiem; K01DK076973 to B.C. Beard; R01CA164371, R01NS060752, and U54CA143970 to K.R. Swanson). H.-P. Kiem is a Markey Molecular Medicine Investigator and received support as the inaugural recipient of the José Carreras/E. Donnall Thomas Endowed Chair for Cancer Research. K.R. Swanson is a Robert H. Lurie Comprehensive Cancer Center Investigator at Northwestern University. K.R. Swanson was a recipient of the James D. Murray Endowed Chair and Academic Pathology Fund, both at UW, and Zell Scholar and Wirtz Scholar awards at Northwestern University. K.R. Swanson is a recipient of the James S. McDonnell Foundation award.

Address correspondence to: Hans-Peter Kiem, Fred Hutchinson Cancer Research Center, Mail Stop D1-100, P.O. Box 19024, Seattle, Washington 98109-1024, USA. Phone: 206.667.4425; E-mail: hkiem@fhcrc.org. 
1. Tanaka S, Louis DN, Curry WT, Batchelor TT, Dietrich J. Diagnostic and therapeutic avenues for glioblastoma: no longer a dead end? Nat Rev Clin Oncol. 2013;10(1):14-26.

2. Stupp R, et al. Effects of radiotherapy with concomitant and adjuvant temozolomide versus radiotherapy alone on survival in glioblastoma in a randomised phase III study: 5-year analysis of the EORTC-NCIC trial. Lancet Oncol. 2009;10(5):459-466.

3. Stupp R, et al. Radiotherapy plus concomitant and adjuvant temozolomide for glioblastoma. N Engl J Med. 2005;352(10):987-996.

4. Liu L, Gerson SL. Targeted modulation of MGMT: clinical implications (Review). Clin Cancer Res. 2006;12(2):328-331.

5. Quinn JA, et al. Phase I trial of temozolomide plus O6-benzylguanine 5-day regimen with recurrent malignant glioma. Neuro Oncol. 2009;11(5):556-561.

6. Quinn JA, et al. Phase II trial of temozolomide plus o6-benzylguanine in adults with recurrent, temozolomide-resistant malignant glioma. J Clin Oncol. 2009;27(8):1262-1267.

7. Allay JA, Dumenco LL, Koc ON, Liu L, Gerson SL. Retroviral transduction and expression of the human alkyltransferase cDNA provides nitrosourea resistance to hematopoietic cells. Blood. 1995;85(11):3342-3351.

8. Beard BC, Trobridge GD, Ironside C, McCune JS, Adair JE, Kiem H-P. Efficient and stable MGMTmediated selection of long-term repopulating stem cells in nonhuman primates. J Clin Invest. 2010;120(7):2345-2354.

9. Adair JE, et al. Extended survival of glioblastoma patients after chemoprotective HSC gene therapy. Sci Transl Med. 2012;4(133):133ra57.

10. Li J, et al. Validation and simplification of the
Radiation Therapy Oncology Group recursive partitioning analysis classification for glioblastoma. Int J Radiat Oncol Biol Phys. 2011;81(3):623-630.

11. Wen PY, et al. Updated response assessment criteria for high-grade gliomas: response assessment in neuro-oncology working group. J Clin Oncol. 2010;28(11):1963-1072.

12. Neal ML, et al. Discriminating time to progression and survival using a response metric tuned to patient-specific glioblastoma kinetics. PLoS One. 2013;8(1):e51951.

13. Neal ML, et al. Resonse classification based on a minimal model of glioblastoma growth is prognostic for clinical outcomes and distinguishes progression from pseudoprogression. Cancer Res. 2013;73(10):2976-2986.

14. Karnofsky DA, Burchenal JH. The clinical evaluation of chemotherapeutic agents in cancer. In: Macleod CM, ed. Evaluation of Chemotherapeutic Agents. New York, NY: Columbia University Press; 1949:191-205.

15. Yin AA, et al. The predictive but not prognostic value of MGMT promoter methylation status in elderly glioblastoma patients: a meta-analysis. PLoS One. 2014;9(1):e85102.

16. Swanson KR, et al. Velocity of radial expansion of contrast-enhancing gliomas and the effectiveness of radiotherapy in individual patients: a proof of principle. Clin Oncol (R Coll Radiol). 2008;20(4):301-308.

17. Swanson KR, Rostomily RC, Alvord EC Jr. A mathematical modelling tool for predicting survival of individual patients following resection of glioblastoma: a proof of principle. Br JCancer. 2008;98(1):113-119.

18. Wang $\mathrm{CH}$, et al. Prognostic significance of growth kinetics in newly diagnosed glioblasto- mas revealed by combining serial imaging with a novel biomathematical model. Cancer Res. 2009;69(23):9133-9140.

19. Swanson KR, Alvord EC Jr, Murray JD. Virtual brain tumours (gliomas) enhance the reality of medical imaging and hilight inadequacies of current therapy. Br J Cancer. 2002;86(1):14-18.

20. Rockne R, et al. Predicting the efficacy of radiotherapy in individual glioblastoma patients in vivo: a mathematical modeling approach. Phys Med Biol. 2010;55(12):3271-3285.

21. Mandonnet E, et al. Continuous growth of mean tumor diameter in a subset of grade II gliomas. Ann Neurol. 2003;53(4):524-528.

22. Beard BC, Mezquita P, Morris JC, Kiem H-P. Efficient transduction and engraftment of G-CSF-mobilized peripheral blood CD34 ${ }^{+}$ cells in nonhuman primates using GALVpseudotyped $\gamma$-retroviral vectors. Mol Ther. 2006;14(2):212-217.

23. Swanson KR, Alvord EC Jr, Murray JD. A quantitative model for differential motility of gliomas in grey and white matter. Cell Prolif. 2000;33(5):317-329.

24. Harpold HL, Alvord EC Jr, Swanson KR. The evolution of mathematical modeling of glioma proliferation and invasion. J Neuropathol Exp Neurol. 2007;66(1):1-9.

25. Szeto MD, et al. Quantitative metrics of net proliferation and invasion link biological aggressiveness assessed by MRI with hypoxia assessed by FMISO-PET in newly diagnosed glioblastomas. Cancer Res. 2009;69(10):4502-4509.

26. Shaw EG, et al. Reexamining the radiation therapy oncology group (RTOG) recursive partitioning analysis (RPA) for glioblastoma multiforme (GVM) patients [abstract]. Int J Radiat Oncol Biol Phys. 2003;57(2, Supplement). 